\title{
Running Variance Metric for evaluating performance of Wireless IP Networks in the MobileCity Testbed
}

\author{
Christer Åhlund \\ Luleå University of \\ Technology, Department \\ of Computer Science, SE- \\ 97187 Luleå, Sweden \\ christer.ahlund@ltu.se
}

\author{
Robert Brännström \\ Luleå University of \\ Technology, Department of \\ Computer Science, SE-971 87 \\ Luleå, Sweden \\ robert.brannstrom@ltu.se
}

\author{
Arkady Zaslavsky \\ School of Computer Science \& \\ Software Engineering, Monash \\ University, \\ 900 Dandenong Road, Caulfield \\ East,
}

Vic 3145, Melbourne, Australia

a.zaslavsky@csse.monash.edu.au

\begin{abstract}
This paper proposes and analyzes a Running Variance Metric performance measurement of wireless local area networks and its formal aspects. Our approach evaluates the performance of wireless local area networks in infrastructure mode as well as in ad hoc mode. The Running Variance Metric is used to discover relative traffic loads of available accesspoints/gateways at the network layer in order to provide connectivity to the wired network. The paper discusses a simulation study. The simulation results demonstrate the usefulness and efficiency of the Running Variance Metric to evaluate the utilization of available access-points/gateways. It is also shown that this metric can be used for hop-analysis in multi-hop ad hoc wireless networks.
\end{abstract}

\section{Introduction}

This article proposes and discusses an approach to evaluate the relative traffic load at the network layer when connecting to access points (AP) used in infrastructure networks and gateways connecting between wired IP networks and ad hoc networks. This is useful for a mobile host (MH) using Mobile IP (MIP) [1] and for Global Connectivity [2] during handover or when being multihomed and selecting the AP/gateway to use. When using MIP with infrastructure networks, the $\mathrm{MH}$ has to rely on the datalink layer to make a good decision on which AP to use if multiple APs are available. After associating with the AP, the network layer is able to discover the network connecting the AP and register according to MIP. The decision made at the datalink layer may not be optimal considering the performance based on throughput. To enable this there is a need to discover the network layer performance when deciding which AP to use. With ad hoc networks connectivity to gateways connecting to wired IP networks also needs a way to decide which gateway to use. Proposals given for this are usually based on the hop-count as described in [3]. Another solution is presented in [4]. However, a dynamic metric reflecting the utilization will be beneficial for this decision.

\subsection{Infrastructure Networks}

MHs when connecting to an AP make decisions based on the signal-to-noise ratio (SNR) and related factors. This information originates at the physical layer and is analyzed at the datalink layer in the IPstack. However, SNR does not reflect the performance of the AP at the network layer. This means that calculating the SNR values will not be enough to decide the best AP to associate with considering the throughput. In some situations a better throughput can be achieved by using APs with lower SNR values. With the same SNR the throughput may also differ. According to the 802.11 [5] standard, MH3 in figure 1 may associate with AP1 even though more traffic is sent by MH1 and MH2 than by MH4 and MH5. Or, in other words, AP2 is carrying less traffic that AP1. As illustrated by the left circle, MH3 is out of communication range from $\mathrm{MH} 1$ and $\mathrm{MH} 2$, and cannot detect collisions generated by these nodes in the SNR calculation. In 802.11 there is also a Network Allocation Vector (NAV) that is used by a sender to signal the time needed to send a frame. With the usage of NAV fewer collisions will occur. So it is clear that the SNR is not appropriate to use as the only metric when deciding which AP to use. 


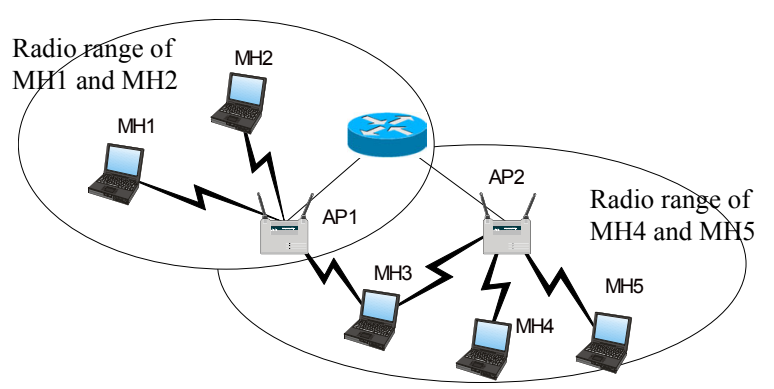

Figure 1. A sample topology.

For infrastructure Wireless Local Area Networks (WLANs), calculations based on measurements at the network layer can be used to decide which AP to use if multiple APs are available.

\subsection{Ad Hoc Networks}

For ad hoc networks where gateway connectivity to the wired network is required, the network layer performance should be used both when multiple gateways are available as well as when an $\mathrm{MH}$ has multiple paths to a gateway.

In existing networks with today's traffic pattern, most network traffic is to destinations outside a LAN. The 20/80 ratio used to classify today's network traffic indicates that $20 \%$ of the network traffic is within that LAN, and $80 \%$ of the traffic is to destinations outside the LAN. This means that $80 \%$ of the traffic has to go through the gateway.

In our model, we consider ad hoc networks as subnetworks [6], and that services like the Domain Name Service (DNS), Dynamic Host Configuration Protocol (DHCP) remain external to the ad hoc networks. This is due to the fact that MHs are mobile with a high probability of moving to other networks. However, there is ongoing research on how to support these services inside ad hoc networks, for example, DNS services in ad hoc networks [7].

Based on these observations, maintaining connectivity to gateways is important, and choosing the one with the best performance will improve the throughput. The routing protocols proposed for ad hoc networks (e.g. DSR [8], AODV [9]) usually assume the same capacity for all links across the network, and use the hop count as the routing metric. Therefore a 2 hop route will be preferred over a 3 hop route despite the utilization of links. Even though the 2 hop route carries more traffic than the 3 hop route it will be selected. Ad hoc routing protocols that are considering only the hop count will face the same problems as RIP version 1 does in wired IP networks. Dynamic metrics need to be proposed and applied to ad hoc networks to overcome these problems. In this paper we limit the scope of dynamic metrics to gateway connectivity only.

We propose a complementary metric that will enable an $\mathrm{MH}$ to evaluate the performance of a wireless link at the network layer and to choose the AP which provides the best throughput.

This paper is structured in the following way. Section 2 describes the formal reasoning used to calculate the Running Variance Metric. Section 3 describes a simulation model and the results of that simulation. In section 4 a description of how our algorithm will be used in a testbed is presented. Section 5 describes related works and section 6 concludes the paper.

\section{Running Variance Metric}

To evaluate the relative traffic load of available APs/gateways we use periodical advertisements sent by them. These advertisements can be router advertisements [10] (available in IP version 4 (IPv4) and IP version 6 (IPv6)) or agent advertisements in MIP version 4 (MIPv4). In MIP version 6 (MIPv6), the router advertisement in IPv6 is used. With increased traffic the AP/gateway may not cope with incoming and out-going traffic. This will lead to buffering of advertisements and collisions between advertisements and traffic. If the send buffer at an AP/gateway is full, some advertisements will be dropped. When the link becomes less congested two or more advertisements could be sent in more dense succession. This, in turn, means that with increased traffic the arrival times of advertisements at MHs will vary. Collisions of advertisements also affects the arrival times, since these advertisements are destroyed and do not arrive at MHs. We make use of the variance in arrival times of advertisements to evaluate the degree of links load. The following formulas introduce the variance metric.

Formula 1 calculates the mean value of the time between arrivals of advertisements and is based on the formula for weighted mean $\left(\bar{x}_{n}\right)$ values [11]. Formula 2 then calculates the variance $\left(V_{n}\right)$ of the arrived advertisements and this is used for the evaluation of wireless links. The variable $t_{n}$ is the arrival time of the last advertisement, $t_{n-1}$ is the arrival time of the previous advertisement. The variable $n$ symbolizes the number of advertisements received since the $\mathrm{MH}$ started to receive advertisements from an AP/gateway. With the variable $h$ we select a history window 
expressing how long history to consider when calculating the mean value and variance.

$\bar{x}_{n}=\frac{1}{h} \delta_{n}+\frac{h-1}{h} \bar{x}_{n-1}$

$V_{n}=\frac{1}{h}\left(\delta_{n}-\bar{x}_{n}\right)^{2}+\frac{h-1}{h} * V_{n-1}$

The variables $h, \bar{x}_{0}$ and $V_{0}$ is initialized with the following values:

$\frac{1}{h}=\{z: z>0 \wedge z \leq 1\}$
$V_{0}=0$
$\bar{x}_{0}=$ Defined advertisement time

The variable $\delta_{n}$ is calculated as:

$\delta_{n}=\left\{t_{n}-t_{n-1}: n>0\right\}$

Formula 2 is an approximation of the mathematically defined variance and is shown by:

$$
\begin{aligned}
& V_{n}=\frac{1}{n} \sum_{i=1}^{n}\left(\delta_{i}-\bar{x}_{n}\right)^{2}=\frac{1}{n}\left[\left(\delta_{n}-\bar{x}_{n}\right)^{2}+\sum_{i=1}^{n-1}\left(\delta_{i}-\bar{x}_{n}\right)^{2}\right]= \\
& =\frac{1}{n}\left[\left(\delta_{n}-\bar{x}_{n}\right)^{2}+(n-1) \frac{1}{(n-1)} \sum_{i=1}^{n-1}\left(\delta_{i}-\bar{x}_{n}\right)^{2}\right]
\end{aligned}
$$

We put $V_{n-1}=\frac{1}{(n-1)} \sum_{i=1}^{n-1}\left(\delta_{i}-\bar{x}_{n}\right)^{2} \Rightarrow$

$$
\begin{gathered}
V_{n}=\frac{1}{n}\left[\left(\delta_{n}-\bar{x}_{n}\right)^{2}+(n-1) V_{n-1}\right] \\
\because V_{n}=\frac{1}{n}\left(\delta_{n}-\bar{x}_{n}\right)^{2}+\frac{n-1}{n} V_{n-1}
\end{gathered}
$$

The approximation is created by $V_{n-1}=\frac{1}{(n-1)} \sum_{i=1}^{n-1}\left(\delta_{i}-\bar{x}_{n}\right)^{2}$ where $\bar{x}_{n}$ includes $\delta_{n}$. The previous variance would not include $\delta_{n}$, only $\delta_{l}$ to $\delta_{n-1}$ is included for the "true" variance in the mean value. We will refer to our calculation of the variance as the "Running Variance Metric" (RVM) in the rest of the paper. Next section will discuss the simulation study based on the RVM.

\section{RVM Simulations study}

This section evaluates the RVM calculation and how RVM is applied in the analysis of wireless links in infrastructure mode and in independent BSS mode (ad hoc mode). Our simulation study uses the GlomoSim simulation model version 2.4 [12].

Simulation study results are presented in figures 2 , 4, 5, 7 and 9. The graphs with error bars represent the mean value of multiple simulations (different seeds) using a confidence interval of $95 \%$. Our simulation study has selected two packet sizes based on the publications $[13,14]$. In [13] it is stated that the major parts $(50 \%)$ of the packets have the size of the Maximum Transmission Unit (MTU). We choose an MTU of 1500 bytes in the simulation, being the MTU of Ethernet. The second most widely used MTU is 576 bytes [14]. Packets about this size are, except for TCP traffic, used for UDP traffic, for example for Voice over IP (VoIP). The advertisements used in the simulations have a size of 32 bytes.

Our simulation first analyzes the difference between the RVM and the "true" variance in the following way. Advertisements are sent every second from an AP with varying load. This load is based on different numbers of MHs communicating through the AP with varying throughput. Figure 2 shows the correlation between the RVM and the "true" variance. The solid green curve plots the "true" variance and the red dotted curve plots the RVM. The figure shows 105 calculations of the variance with 40 to 60 values $\left(\delta_{n}\right)$ in each calculation. The range of values generated by the simulation is between $0.96-5.0$ seconds. The graph shows a good correlation between the RVM and "true" variance. In this simulation we used $h=n$ for this comparison.

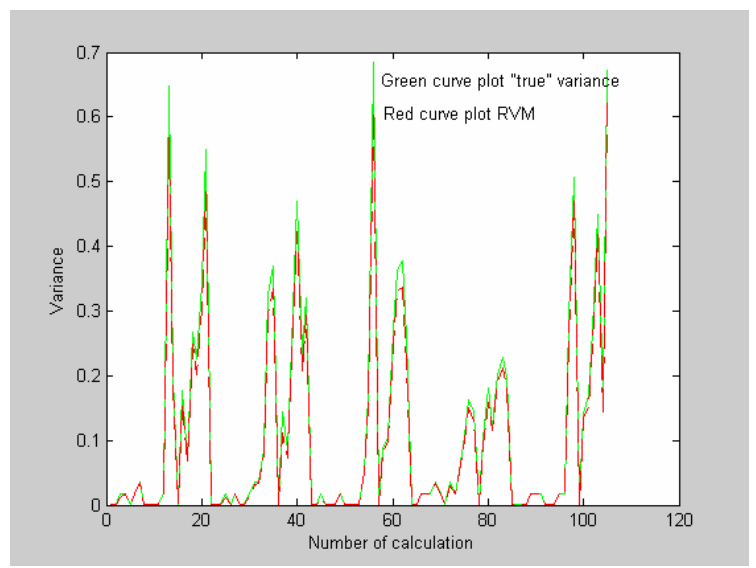

Figure 2. The correlation between the RVM and the "true" variance 


\subsection{Infrastructure Networks}

To demonstrate the RVM's capability for discovering the relative traffic load in wireless infrastructure networks we use the topology shown in figure 3. From one to five MHs send wireless traffic ranging between $0.5 \mathrm{Mbps}$ and $1.5 \mathrm{Mbps}$ with an MTU of 1500 bytes through the AP. The monitoring MH does the RVM calculation. The bandwidth used is defined by $802.11 \mathrm{~b}$ and is $11 \mathrm{Mbps}$.

The results from the simulation are shown in figure 4. The RVM increases with the number of nodes as well as with the amount of traffic sent. Advertisements are sent once every second by the AP. The node monitoring the variance is only within communication range from the AP and not the other nodes.

The plotted lower curve shows the RVM when up to five nodes send $0.5 \mathrm{Mbps}$ each to the AP. The middle curve shows the same for $1 \mathrm{Mbps} / \mathrm{MH}$ and the upper curve shows the RVM for $1.5 \mathrm{Mbps} / \mathrm{MH}$. The RVM demonstrated for one node sending $0.5 \mathrm{Mbps}, 1$ Mbps and 1.5 Mbps is too small to be shown in the graph presented here. However the RVM is doubled for each increased step of the traffic. The big jump of the RVM in the upper and middle curves is explained by the fact that the link is congested, resulting in more collisions.

The same simulation was tried using a transmission unit of 576 bytes. The results are shown in figure 5 . With smaller packets the RVM for $1.0 \mathrm{Mbps}$ and 1.5 Mbps tend to converge near saturation in the wireless link. This is due to small differences in the deviation of advertisements between the two flows when the link is nearly congested. However, for each added node the RVM increases.

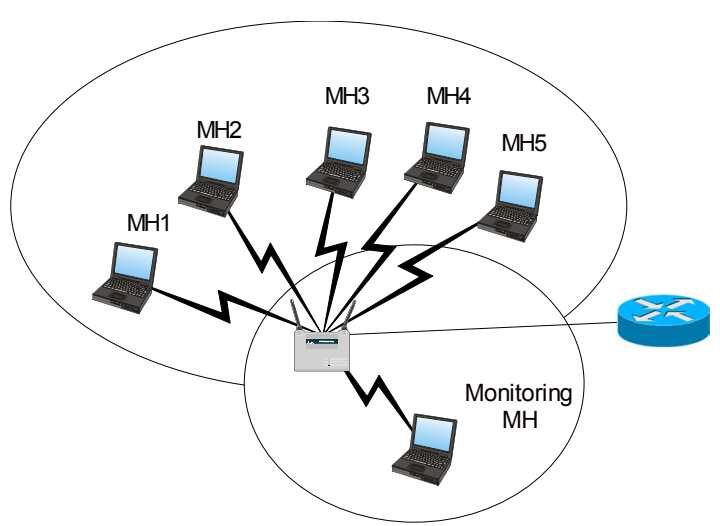

Figure 3. The infrastructure mode topology used in the simulation.
In figure 5 the big jump appears before congestion. This is explained by the increased number of packets sent with a packetsize of 576 bytes compared to a packet size of 1500 bytes. The number of collisions therefore increases, rendering in big contention windows.

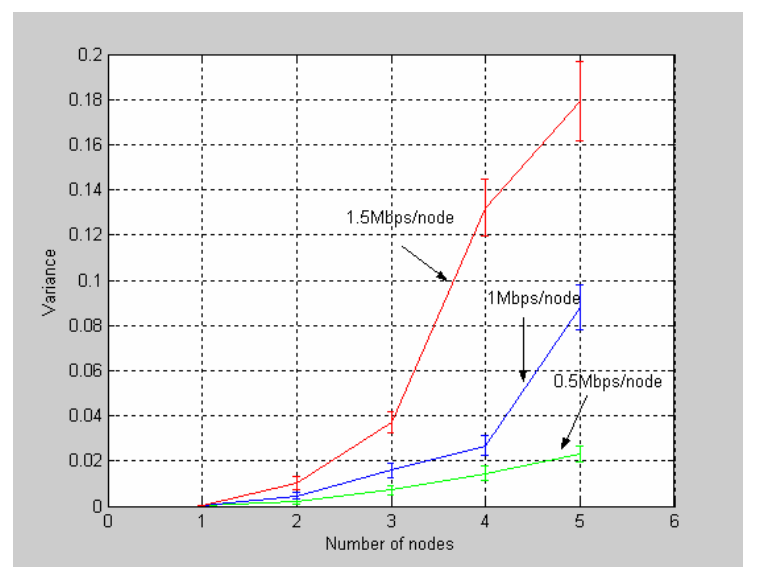

Figure 4. RVM calculations in infrastructure mode, with a packet size of 1500 byte.

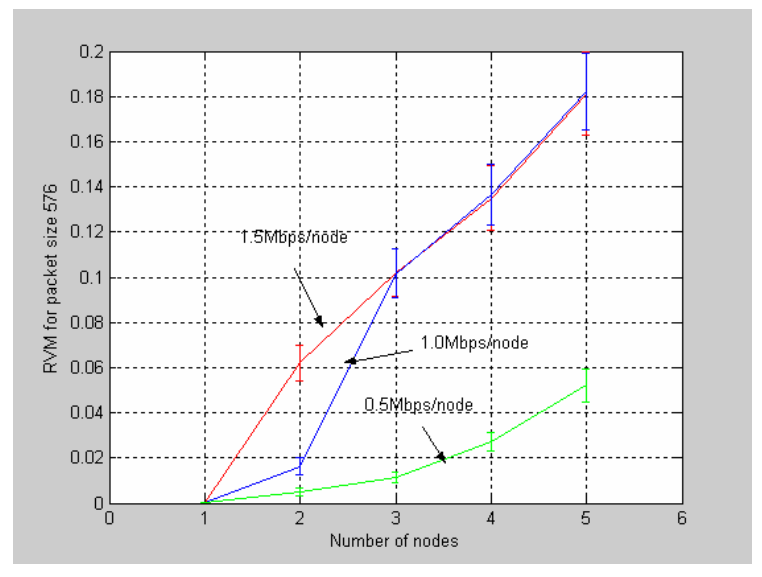

Figure 5. RVM calculations in infrastructure mode, with a packet size of 576 byte.

\subsection{Ad Hoc Networks}

Figure 6 and 7 depicts the wireless multi-hop networks used for the simulations of ad hoc networks. These topologies have been used to evaluate the RVM calculation in ad hoc networks when all wireless links use the same channel.

The simulation study looks at RVM from the view point of differentiation in the number of hops an advertisements travels as well as the utilization of multi hop routes. Every node only sees one or two 
neighbors. A link capacity of $2 \mathrm{Mbps}$ is used in the simulation.

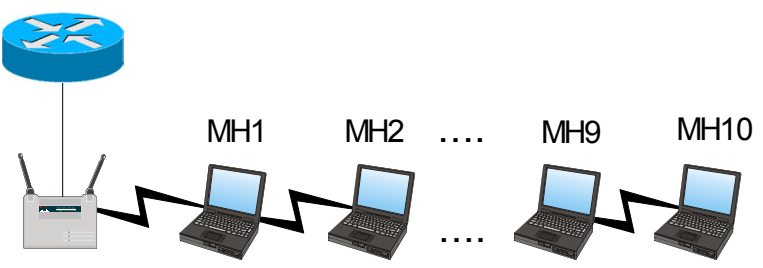

Figure 6. The ad hoc mode topology used in the simulation for calculating RVM at each hop.

The first simulation uses the topology shown in figure 6. Advertisements are sent by the gateway and forwarded from MH1 to MH10. The RVM calculated at each hop is presented in figure 7. As shown, the RVM increases for each hop.

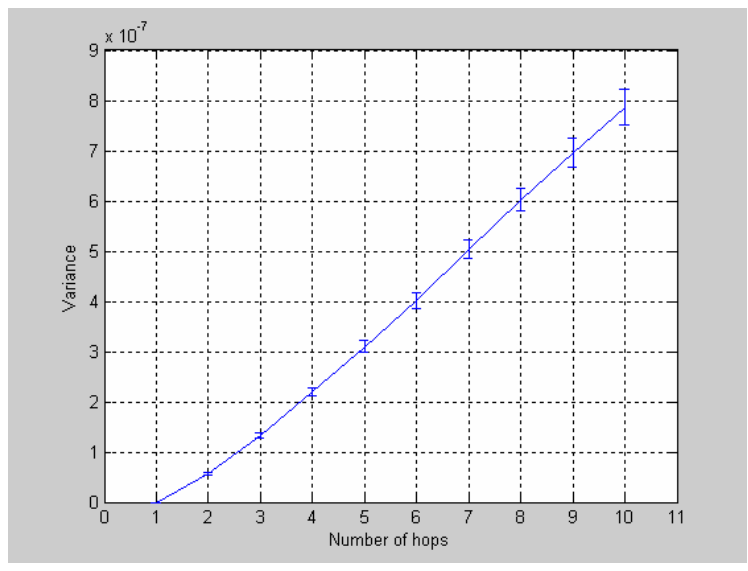

Figure 7. The RVM calculated at each hop.

To see how added traffic flows affect the RVM, we use the topology shown in figure 8 . We monitor the RVM after 5 hops (in MH5) and insert up to four additional 0.5 Mbps flows between MH11 and MH18. The radio ranges of these will only affect MH1 and $\mathrm{MH} 2$.

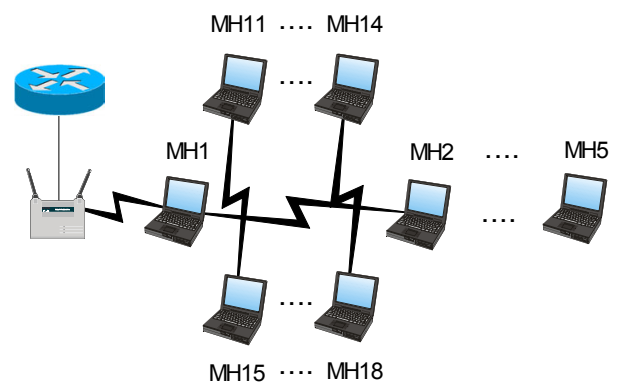

Figure 8. The ad hoc mode topology for RVM monitoring in MH5.
Figure 9 shows results of the simulation where the monitored RVM at MH5 increases for each inserted $0.5 \mathrm{Mbps}$ flow.

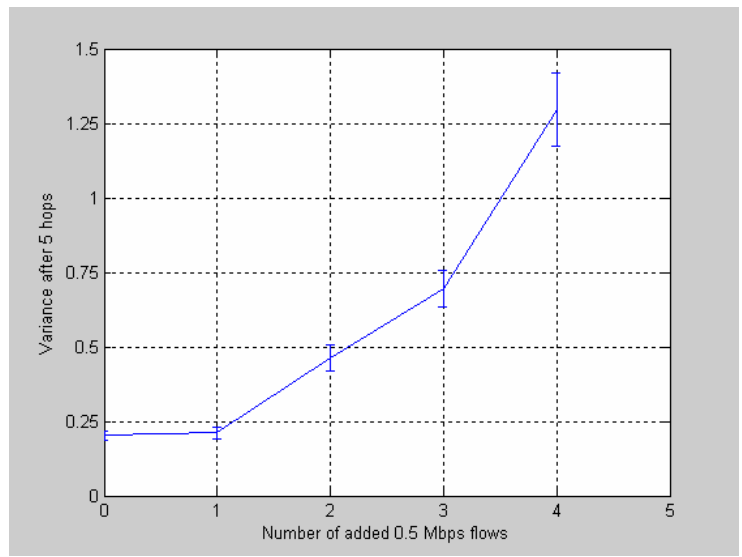

Figure 9. The RVM in MH5.

\section{The testbed implementation}

The testbed that will be used for evaluating a prototype using our algorithm is the MobileCity testbed (www.mobilecity.nu). Within MobileCity a wireless access network based on 802.11 is created. This network spans different locations in the city of Skellefeå in Sweden, including a campus area, city centre, hotels and places visited by tourists. This wireless access network is named SkellefteOpen. This summer of 2004 the testbed was extended with an $802.16 \mathrm{a}$ wireless network covering sectors within and around the city.

A campus building hosting lecturer, researchers and students will be used for our prototype. The APs currently installed and connecting to SkellefteOpen have some dead spots. At the same time people in meeting rooms like to exchange documents, etc. without having to reconfigure devices or depending on an infrastructure.

For this we build a prototype that creates an ad hoc network as a subnetwork and enables connectivity to multiple gateways. Users will be able to communicate peer-to-peer and to use gateways for communication outside the network. If an $\mathrm{MH}$ is in a dead spot intermediate hosts will relay the traffic. With this prototype we will evaluate both infrastructure communication as well as ad hoc communication with our algorithm. Figure 10 shows a possible scenario with our prototype. 


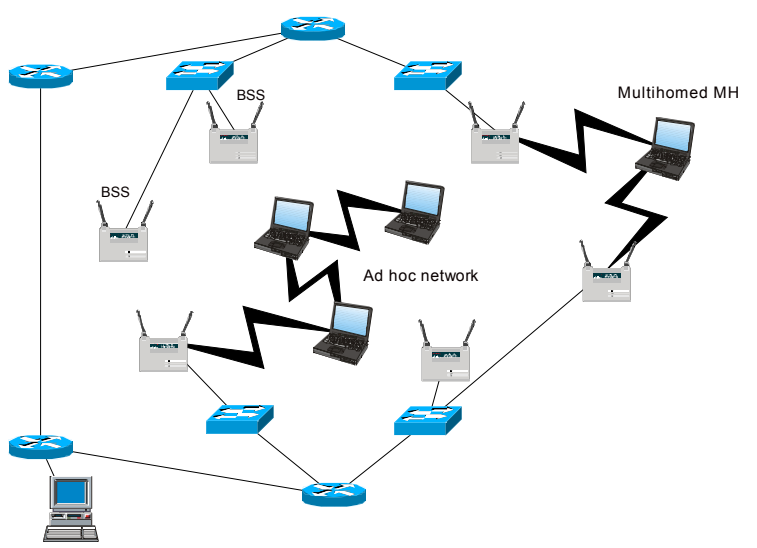

Figure 10. A scenario.

The prototype that we are developing is using the Linux 2.4 kernel [15]. We develop our own Mobile IP implementation extended with multihoming functionality, and we base the ad hoc implementations on AODV-UU [16]. AODV-UU is extended to enable redistribution of MIP information to AODV, so that routes can be created out of this information.

\section{Related work}

With mobility of MHs between WLANs handover is managed both at the datalink layer and the network layer, when MIP is used. The network layer handover can take place only after handover at the datalink layer has been completed. The related work presented here focuses on enhancing the performance of MIP in wireless access networks and to minimize the number of packets lost due to handover. The research described in [17-23] all uses the SNR to decide the AP to associate with. Synchronization is used between the datalink layer and the network layer during handover. A solution enabling an $\mathrm{MH}$ to select the AP based on the utilization is presented in [24].

Methods for horizontal and vertical handoffs are discussed in $[17,23]$. These approaches use multicast to reach multiple nearby APs. MHs instruct APs to forward or buffer data packets for it. If not delivered to the $\mathrm{MH}$, these packets are dropped after some time.

In [18] a proposal is presented to lower the delay with MIP messages and thereby manage handover at the network layer more efficiently, considering the time for handovers. The proposal uses two care-of addresses; link local care-of address (LCoA) and regional care-of address (RCoA). A Mobility Anchor Points (MAP) is used. A MAP manages multiple networks and can be hosted in a gateway connecting an autonomous system (AS). When an MH enters an AS it requires two addresses, LCoA and RCoA. The RCoA address is registered at the home agent (HA) and the LCoA is used for registrations with the MAP. A binding between the RCoA and LCoA is maintained in the MAP. As long at the MH remains within the networks controlled by the MAP the only binding update needed when moving between different networks is the LCoA address sent to the MAP. The registration at the HA remains unaffected.

A solution for fast handover [19] in MIP uses signaling between the $\mathrm{MH}$, the old $\mathrm{AP}$ and the new AP entered to avoid losing packets. Packets will be forwarded from the old to the new AP to avoid packet losses.

Another solution [20] combines the proposals $[18,19]$ and extend it to lessen the handover time even further. The handover time in this work is the same as the handover times for datalink layer handovers. In this proposal the $\mathrm{MH}$ decides when to handover and the network decides where to handover. The network monitors the MHs movement and based on this makes the decision of which AP to use next.

A solution to policy-enabled handoffs is proposed in [23]. This solution is based on three factors: power consumption, cost and bandwidth. The bandwidth usage is monitored and announced by APs so that MHs can calculate the utilization of an AP. This information is used to decide which AP to use.

In [21] a proposal using MIP is given to decrease the time for handover, and to lessen the packet drops. An $\mathrm{MH}$ doing handover at the datalink layer tells the old foreign agent (FA) to buffer packets for it. After the $\mathrm{MH}$ associates with a new FA, the HA tells the old FA to forward buffered packets to the new FA. In the proposal an FA sending agent advertisements includes a neighbor list in the message. The neighbor list includes the IP address, link layer type and channel information of the neighbors. The information is used to enable the $\mathrm{MH}$ to select which FA to handover to. To avoid having to wait for three times the advertisement time (as specified in the MIP specification) to discover loss of connection to a FA, a signal from the datalink level is used to inform the network layer. All agents need to know the position of all neighbor FAs.

In [22] support for fast handover is managed at the datalink level. This proposal is based on the usage of a MAC bridge assisting in bridging packets to a roaming MH's new location, while MIP registration is in process. This avoids losing packets during network layer handover. The delay for handover where packets can be lost only includes the datalink layer handover 
time. This method only works as long as all MHs do handover to APs connected to the MAC bridge. In a real system this is hardly the case, but for micro mobility it can be used.

$[3,4]$ discuss connectivity between wired IP networks and ad hoc networks where MIP is used for mobility between networks. In [3] the hop-count is used for the decision of which FA to use. Handover is trigged when the hop-count to a new FA is two hops less than to the FA currently used. The proposal for gateway selection in [4] uses the following criteria: the $\mathrm{MH}$ has not heard from its FA for at least one advertisement interval, and the MHs route to the FA has become invalid. When this happens handover occurs.

The related work presented addresses how to achieve a more effective handover at the network layer and the decision where to handover both in wireless infrastructure networks and in ad hoc networks. Except for [24] none of the related work addresses network layer performance for this decision. In [24] this is addressed for infrastructure networks but it requires APs to be modified. With our approach we can compare the utilization at the network layer of APs with today's systems, avoiding modifications of APs.

\section{Conclusion}

This paper addresses performance measurement in WLANs. We have proposed and shown how to discover the relative traffic load at MHs in the network layer when connecting wirelessly to APs/gateways. Our methodology uses passive measurements based on advertisements like MIP agent advertisements and router advertisements. The RVM can be used in infrastructure mode as well as in ad hoc mode. With increased traffic on a wireless link, collisions will increase and packets will be delayed in buffers. The simulation study reported in this paper demonstrates that RVM is a complementary metric that can be used in combination with SNR to improve efficiency and throughput of wireless communications between MHs and APs/gateways. This simulation study also supports the theoretical contribution presented in $[2,25]$.

RVM will be used with MIP and Global Connectivity solutions to manage handover and multihoming. We use RVM with Multihomed MIP in [25] to associate with multiple APs. With the proposed approach it is possible to select the least loaded AP(s) when two or more APs are used. No double casting or multicasting is needed because the $\mathrm{MH}$ is connected to multiple APs receiving unique packets. Multiple associations are maintained in order to evaluate the performance of APs. In [2] the RVM is used to evaluate multihop connectivity to gateways in ad hoc networks.

A small "ground" variance should be used for sending advertisements [10], so that a flow (possibly with low utilization of the wireless link) with the same timing as the advertisements does not put out advertisements by colliding with them.

Our approach is currently being implemented in a real system and will be evaluated in the MobileCity testbed (www.mobilecity.nu).

The publication [26] shows how MIP performs using RVM for the selection of FAs

\section{References}

[1] C. Perkins, Mobile IP IEEE Communications Magazine, vol. 40, no. 5, pp. 66-82, May, 2002.

[2] C. Ahlund and A. Zaslavsky, Extending Global IP Connectivity for Ad Hoc Networks Telecommunication Systems, Modeling, Analysis, Design and Management, vol. 24, pp. 221-250, Oct, 2003.

[3] U. Jönsson, f. Alriksson, T. Larsson, P. Johansson, and G.-Q. Maguire, "MIPMANETMobile IP for Mobile Ad Hoc Networks," Mobile and Ad Hoc Networking and Computing, pp. 75-85, Aug. 2000.

[4] Y. Sun, E. M. Belding-Royer, and C. E. Perkins, Internet Connectivity for Ad hoc Mobile Networks International Journal of Wireless Information Networks special issue on 'Mobile ad Hoc Networks (MANETs): Standards, esearch, Applications', vol. 9, pp. 75-88, Apr, 2002.

[5] M. S. Gast. 802.11 Wireless Networks, The Definite Guide, O'Reilly, 2002.

[6] C. Ahlund and A. Zaslavsky, "Software Solutions to Internet Connectivity in Mobile Ad hoc Networks," 4th International Conference on Product Focused Software Process Improvement, pp. 559-572, Dec. 2002. 
[7] P. Engelstad , D.V. Thanh, and T.E. Jonvik, "Name Resolution in Mobile Ad-hoc Networks," 10th International Conference on Telecommunications, Feb. 2003.

[8] D. B. Johnson and D. A. Maltz, Dynamic Source Routing in Ad Hoc Wireless Networks Mobile Computing, vol. 353, pp. 153-181, 1996.

[9] C. E. Perkins and E. M. Belding-Royer, "Adhoc On Demand Distance Vector Routing," 2nd IEEE Workshop on Mobile Computing Systems and Applications, pp. 90-100, Feb. 1999.

[10] S. Deering. ICMP Router Discovery Message. RFC 1256. September 1991.

[11] L. Rade and B. Westergren. Beta Mathematics Handbook, Studentlitteratur, pp. 46

[12] UCLA Parallel Computing Laboratory. Glomosim, http://pcl.cs.ucla.edu/projects/glomosim/. Sept. 2004.

[13] C. Williamson, Internet Traffic Measurement IEEE Internet Computing, vol. Vol 5, pp. 7074, Nov, 2001.

[14] A. Klemm, C. Lindemann, and M. Lohmann, "Traffic Modeling of IP Networks Using the Batch Markovian Arrival Process, Lecture Notes In Computer Science archive," Proceedings of the 12th International Conference on Computer Performance Evaluation, Modelling Techniques and Tools, pp. 92-110.

[15] Linux, http://www.linux.org. Sept. 2004.

[16] AODV-UU, http://user.it.uu.se/ henrikl/aodv/. Sept. 2004.

[17] M. Stemm and R. H. Katz, Vertical Handoffs in Wireless Overlay Networks Mobile Networks and Applications, vol. 3, pp. 335-350, 1998.

[18] H. Soliman et al. Hierarchical MIPv6 mobility management. 2004. Internet Draft, IETF.
[19] G. Dommety, K.-E. Malki, M. Khalil, C. Pergins, H. Soliman, G. Tsirtsis, and A.-E. Yegin . Fast Handover for Mobile IPv6. 2004. Internet Draft, IETF.

[20] R. Hseih, Z.-G. Zhou, and A. Seneviratne, "SMIP: A Seamless Handoff Architecture for Mobile IP," pp. 1774-1784, Apr. 2003.

[21] J. C.-S. Wu , C.-W. Cheng, N. -F. Huang, and G. -K. Ma, Intelligent Handoff for Mobile Wireless Internet Mobile Networks and Applications, vol. 6, pp. 67-79, Jan, 2001.

[22] H. Yokota, A. Idoue, T. Hasegawa, and T. Katao, "Link Layer Assisted Mobile IP Fast Handoff Method over Wireless LAN Networks," 8th International Conference on Mobile Computing and Networking, pp. 131139, Sept. 2002.

[23] S. Seshan, H. Balakrishnan, and R. Katz, Handoffs in Cellular Wireless Networks: The Daedalus Implementation and Experience Wireless Personal Computing, vol. 4, pp. 141162, 1997.

[24] H. J. Wang, R. H. Katz, and J. Giese, "Policyenabled Handoffs Across Heterogeneous Wireless Networks," Proceedings of the Second IEEE Workshop on Mobile Computer Systems and Applications, pp. 51-60, Feb. 1999.

[25] C. Ahlund and A. Zaslavsky, "Multihoming with Mobile IP," 6th IEEE International Conference on High Speed Networks and Multimedia Communications, pp. 235-243, July 2003.

[26] C. Ahlund, R. Brannstrom, and A. Zaslavsky, "M-MIP: extended Mobile IP to maintain multiple connections to overlapping wireless access networks ," International Conference on Networking, Apr. 2005. 